Check for updates

Cite this: RSC Adv., 2019, 9, 31150

Received 3rd July 2019

Accepted 12th September 2019

DOI: $10.1039 / c 9 r a 05032 a$

rsc.li/rsc-advances

\section{A proposed protocol based on integrative metabonomics analysis for the rapid detection and mechanistic understanding of sulfur fumigation of Chinese herbal medicines $\uparrow$}

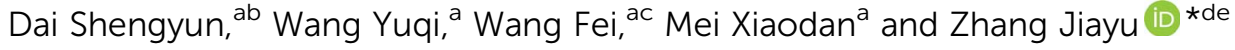

In the current work, Lonicera japonica Flos (FLJ) was selected as a model Chinese herbal medicine (CHM) and a protocol was proposed for the rapid detection of sulfur-fumigated (SF) CHMs. A multiple metabonomics analysis was conducted using HPLC, NIR spectroscopy and a UHPLC-LTQ-Orbitrap mass spectrometer. First, the group discriminatory potential of each technique was respectively investigated based on PCA. Then, the effect of mid-level metabonomics data fusion on sample spatial distribution was evaluated based on data obtained using the above three technologies. Furthermore, based on the acquired HRMS data, 76 markers discriminating SF from non-sulfur-fumigated (NSF) CHMs were observed and 49 of them were eventually characterized. Moreover, NIR absorptions of 18 sulfurcontaining markers were identified to be in close correlation with the discriminatory NIR wavebands. In conclusion, the proposed protocol based on integrative metabonomics analysis that we established for the rapid detection and mechanistic explanation of the sulfur fumigation of CHMs was able to achieve variable selection, enhance group separation and reveal the intrinsic mechanism of the sulfur fumigation of $\mathrm{CHMS}$
\end{abstract}

\section{Introduction}

Sulfur fumigation is a traditional storage method for Chinese herbal medicines (CHMs). It was first applied to the processing of Dioscoreae rhizoma, and has been widely misused in various CHMs in the last two decades, such as in the processing of Chrysanthemi Flos, Gastrodia Rhizoma, Radix Paeoniae Alba and Lonicera japonica Flos (FLJ). ${ }^{1-3}$ It plays an important role in the production and post-harvest handling of CHMs due to its usage in moisturizing, bleaching, retaining freshness and killing parasites. $^{4-6}$ However, sulfur fumigation induces significant chemical transformations in inherent herbal constituents, resulting in alterations in the bioactivities, pharmacokinetics and toxicities of CHMs. ${ }^{7,8}$ Besides, it often leads to the production in CHMs of excessive sulfur dioxide $\left(\mathrm{SO}_{2}\right)$, sulfates, sulfites, heavy metal residues and other detrimental exogenous

\footnotetext{
${ }^{a}$ School of Chinese Pharmacy, Beijing University of Chinese Medicine, Beijing 102488, China

${ }^{b}$ National Institute of Food and Drug Control, Beijing 100050, China

${ }^{c}$ Department of Pharmacy, People Hospital of Peking University, Beijing 100044, China ${ }^{d}$ Beijing Research Institute of Chinese Medicine, Beijing University of Chinese Medicine, Beijing 100029, China. E-mail: zhangjiayu0615@163.com ${ }^{e}$ School of Pharmacy, Binzhou Medical University, Yantai 264003, China

$\dagger$ Electronic supplementary information (ESI) available. See DOI: 10.1039/c9ra05032a
}

materials, which exhibit harmful potential toxicities or side effects to human health. ${ }^{9-11}$

Although the use of sulfur fumigation has been officially restricted in China since $2005,{ }^{12}$ some illicit herbal farmers and wholesalers still misuse sulfur fumigation during the postharvest handling and storage of CHMs. Moreover, $\mathrm{SO}_{2}$ residue-based detection standards formulated by many countries and organizations are often ineffective at evaluating the degree of sulfur fumigation because of the high volatility of $\mathrm{SO}_{2}$. Current studies mainly focus on total $\mathrm{SO}_{2}$ residues and neglect the transformations of inherent herbal constituents and corresponding mechanisms. ${ }^{13-16}$ Therefore, the development of rapid and sensitive approaches based on stable quality-markers (Qmarkers), such as sulfur-containing derivatives, to discriminate sulfur-fumigated (SF) CHMs from non-sulfur-fumigated (NSF) CHMs is urgently needed. ${ }^{17}$

Integrative omics combining and interpreting data from multiple sources have already been adopted to successfully elucidate the mechanisms of human diseases, such as diabetes, obesity and schizophrenia. ${ }^{\mathbf{1 8 , 1 9}}$ Besides, integrative omics analysis has been used to characterize genes in the context of the molecular pathophysiology of the disease and its interacting genes and pathways. ${ }^{\mathbf{2 0 , 2 1}}$ Likewise, multi-omics data collected using various detection technologies such as liquid chromatography combined with mass spectrometry (LC-MS), highperformance liquid chromatography (HPLC) and near infrared 
(NIR) spectroscopy have been used for the screening and identification of Q-markers for the analysis of CHMs. ${ }^{22,23}$ Of these technologies, HPLC retains the practicality and principles of LC, while increasing the overall interlaced attributes of sensitivity and resolution, MS has emerged as a powerful tool for quantitative and qualitative analysis of the complex components in CHMs, and NIR spectroscopy is a very rapid and alternative nondestructive method that shows electromagnetic absorption signals in the NIR region associated with specific chemical structures and that can be assigned to specific chemical functional groups and molecular structures. Nevertheless, although each technique has its own powerful capabilities for specific issues, any data set obtained by one single technique cannot capture the complexity of the overall system. Thus, integrative metabonomics analysis based on multiple levels of data fusion and correlation combines the information provided by various analytical technologies so as to achieve much better statistical predictions and interpretations than those obtained from any individual technique.

FLJ, also known as Jin Yin Hua in Chinese, is one of the most well-known CHMs. It is derived from the dried buds or flowers of Lonicera japonica Thunb. and contains various biological ingredients such as organic acids, flavonoids and iridoid glycosides. $^{24-27}$ Pharmacological investigations indicated that FLJ displays various pharmacological activities, such as hepatoprotective, cytoprotective, anti-microbial, anti-oxidative, anti-viral and anti-inflammatory activities. ${ }^{28-30} \mathrm{FLJ}$ is also used in many food products, such as FLJ tea, a well-known health drink that has been highly praised for thousands of years for clearing away heat and toxic materials and treating exogenous pathogenic wind-heat. ${ }^{31}$ However, in the last two decades, sulfur fumigation has been frequently misused in post-harvest handling during the drying and storage of FLJ. Therefore, we used FLJ as a study case to present a proposal for a protocol based on integrative metabonomics analysis in order to clarify the inherent chemical transformations of CHMs and to classify the CHMs based on these transformations. SF and non-sulfurfumigated (NSF) FLJ along with organic acids, flavonoids and iridoid glycosides were used to verify the effectiveness of the established strategy.

\section{Materials and methods}

\subsection{Chemicals, reagents and herbal materials}

A total of 22 batches of authenticated NSF FLJ samples were collected from several different areas in China (Table S1 $\dagger$ ). All of their identities were authenticated using morphological and histological methods to be the dried buds of $L$. japonica Thunb. according to the monograph of Chinese Pharmacopoeia (version 2015). ${ }^{32}$ The authenticated specimens were deposited in the Beijing Research Institute of Chinese Medicine, Beijing University of Chinese Medicine, China. Standard substances including 3-caffeoylquinic acid (3-CQA), 4-CQA, 5-CQA, 3,4dicaffeoylquinic acid (3,4-DiCQA), 3,5-diCQA, 4,5-diCQA, lonicerin, secologanic acid, swertiamarin and luteolin 7-O- $\beta$-glucoside were purchased from Chengdu Bio-purify Phytochemicals Ltd (Sichuan, China) with their purities all greater than $98 \%$
(Fig. S1 and Table S2 $\uparrow$ ). HPLC-grade acetonitrile and methanol were provided by Fisher Scientific (Fisher, Fair Lawn, NJ, USA). Formic acid was provided by Aldrich (St. Louis, MO). All of the other chemicals were of analytical grade and obtained commercially from Beijing Chemical Works (Beijing, China). De-ionized water was purified using a Milli-Q Gradient A 10 System (Millipore, Billerica, MA). The $0.22 \mu \mathrm{m}$ membranes were purchased from Xinjinghua Co. (Shanghai, China).

\subsection{Sample preparation}

2.2.1 SF FLJ herbal samples. Fifteen batches of SF FLJ samples were prepared following the modified procedures that were employed by farmers and illicit wholesalers. ${ }^{33,34}$ A total of $200 \mathrm{~g}$ of FLJ dried buds were wetted with water and allowed to stand for $0.5 \mathrm{~h}$. Afterwards, a proper amount of sulfur powder was heated until it burned, and then the burning sulfur and wetted FLJ were carefully put into, respectively, the lower and upper layers of a desiccator. The desiccator was then kept closed for $12 \mathrm{~h}$ in order to achieve a sufficient sulfur fumigation. Meanwhile, the SF 5-CQA that was utilized for result validation was also prepared with this same method.

2.2.2 Reference solutions. A certain amount of each of 3CQA, 4-CQA, 5-CQA, 3,4-CQA, 3,5-diCQA, 4,5-diCQA, lonicerin, secologanic acid, swertiamarin and luteolin 7-O- $\beta$-glucoside was respectively weighed accurately and then dissolved in methanol to obtain the mixed reference solutions $(0.01 \mathrm{mg}$ $\left.\mathrm{mL}^{-1}\right)$. These reference solutions were stored at $4{ }^{\circ} \mathrm{C}$ prior to analysis.

2.2.3 FLJ sample solutions. FLJ powders ground and sieved through a 65 mesh sieve were soaked for $30 \mathrm{~min}$. A total of $1.0 \mathrm{~g}$ of powder was accurately weighed and then extracted with $25 \mathrm{~mL}$ methanol/water $(70: 30, \mathrm{v} / \mathrm{v})$ in an ultrasonic bath (40 $\mathrm{kHz}$, Eima Ultrasonics Corp., Germany) for $30 \mathrm{~min}$ at room temperature. Then the same solvent was added to compensate for the lost weight during the extraction process. The methanol solution was subjected to centrifugation (10000 g) for 10 minutes, and then filtered through a $0.22 \mu \mathrm{m}$ microporous membrane before being injected into an LC-MS system for analysis. To ensure the quality of the HPLC and LC-MS-based metabonomics data, pooled quality control (QC) samples were prepared by mixing equal amounts of 37 sample solutions.

NIR, HPLC-DAD and UHPLC-LTQ-Orbitrap MS data were collected from these samples. The conditions for the three methods are listed in the ESI. $\dagger$

\subsection{Primary metabonomics data processing}

All of the data were subjected to principal component analysis (PCA) and partial least squares discriminant analysis (PLS-DA) to interpret the interrelationships between the samples. With respect to PLS-DA, samples were divided into a calibration set for modeling and a validation set for the established model evaluation. The prediction set consisted of samples that were not used for the calibration set.

The very high quantities of acquired UHPLC-LTQ-Orbitrap MS raw data were processed with an Xcalibur 2.1 workstation (Thermo Scientific, Germany). The normalization was 
accomplished using Sieve 2.1 software (Thermo Scientific, USA), which was specifically used to perform background subtraction, component detection and peak alignment. SIMCA-P+ 11.5 (Umetrics, Sweden) and Unscrambler 7.0 (CAMO, Norway) software were utilized to carry out the spectral pre-processing. PCA and PLS were conducted using Matlab version R2009a (The MathWorks, Inc., USA) with Statistical Toolbox and inhouse functions. The iToolbox utilized to run synergy interval partial least squares (siPLS) analysis algorithms was downloaded from http://www.models.kvl.dk/ for the NIR wavelength selection.

\subsection{Integrative metabonomics analysis}

2.4.1 Metabonomics data fusion. In order to integrate the information acquired from the different technologies, mid-level metabonomics data fusion was carried out to investigate the three obtained metabonomics data sets based on NIR, HPLC and LC-MS approaches. Much more attention was paid to the comparison between two methods, i.e., metabonomics data fusion with or without variable selection. The first method specifically applied PCA to describe the metabonomics data and determine the data structure without variable selection. To overcome the shortage of differences in scores and eigenvalues, all of them were standardized and eigenvalues were converted into percentage of explained variance. Scores were then multiplied by eigenvalues, and matrices were combined (HPLC, NIR and HRMS). The second method was based on the selection of relevant variables for each analytical method using PLS-DA with variable selection, which was then investigated using PCA. In this way, the number of explanatory variables was markedly reduced, and the selected variables only described the primary differences between all of the samples.

2.4.2 Method validation. To ensure the reliability of the experimental results, the reference standard, 5-CQA, was obtained to perform the SF process. SF chlorogenic acid samples $(0.5,1.0,2.0,2.5$ and $5.0 \mathrm{mg})$ were respectively weighed, and each sample was then thoroughly mixed with $5.0 \mathrm{mg}$ of dextrin. Meanwhile, NSF chlorogenic acid samples were prepared in the same way to test the reliability of the results. These ten samples were analyzed using the same NIR and LC-MS methods.

\section{Results and discussion}

\subsection{Basic data sets for the three technologies}

Typical NIR, HPLC-DAD and total ion chromatogram (TIC) results of the representative SF and NSF samples are presented in Fig. S2. $\dagger$ After peak alignment and removal of the missing values, 90 features were finally obtained for the HPLC-based metabonomics analysis. Meanwhile, for each of 37 samples, the NIR and MS data sets included 1557 and 5000 variables, respectively.

\subsection{Primary metabonomics data analysis}

Primary data analysis results were obtained respectively from unsupervised PCA and supervised PLS-DA for the three different techniques (Table 1 and Fig. 1).
As for PCA, SF and NSF FLJ samples were not explicitly clustered into two groups with regards to HPLC-DAD and NIR analysis (The preprocess method was SG9 $+2^{\text {nd }}$, and the results obtained from the other preprocess methods are illustrated in Fig. S3 and Table S3 $\dagger$ ). While for the LC-MS analysis results, the 37 SF and NSF samples did segregate into two distinct groups. Fig. 1A-C show plots of these scores for these three techniques, respectively. A distinct classification trend could be observed in the LC-MS score plot. However, the results for these samples were nevertheless scattered considerably, with that for sample number 16 attributed to the NSF group being located in the SF group, which indicated that some of the variation in the samples cannot be obtained from the PCA.

Therefore, PLS-DA was performed to improve the group separation. The PLS-DA model resulted in a clear separation of the SF and NSF samples for each of the three different technologies (Fig. 1D-F). As for the HPLC and LC-MS analyses, statistical models were considered to be statistically significant when the corresponding $Q^{2}$-intercept values $(-0.262$ and $-0.264)$ for the permutation model were negative. Meanwhile, the permuted $R^{2}$ values $(0.624$ and 0.439$)$ were lower than the original $R^{2}$-values (0.903 and 0.927$)$. Additionally, analysis of variance of the cross-validated predictive residuals (CV-ANOVA) tests were performed to confirm that the SF and NSF groups discriminated by PLS-DA were significantly different. The common practice was to interpret a $p$ value $\left(1.16 \times 10^{-5}\right.$ and $3.73 \times 10^{-11}$ ) dramatically lower than 0.05 as contributing a significant model. As for the NIR analysis (for which the preprocess method was SG9+2 ${ }^{\text {nd }}$, and the results obtained from the other preprocess methods are shown in Fig. S4 $\dagger$ ), the $Q^{2}$ intercept value was lower than 0.5 , indicating the poor predictive capability of PLS-DA here. The poor predictive capability was also verified by the finding of a positive $Q^{2}$-intercept value from the permutation test. Therefore, the use of the NIR and HPLC technologies did not achieve a satisfactory classification based on PCA while the use of MS did so.

\subsection{Integrative metabonomics analysis}

3.3.1 Mid-level metabonomics data fusion analysis without variable selection. Data fusion is defined as the integration of data blocks from different analytical platforms into a single model so as to improve the capability of statistical prediction and facilitate interpretation. ${ }^{35}$ Low-level, mid-level and highlevel data fusion have been three commonly used strategies. As the most commonly used strategy, mid-level data fusion can either combine variables after a relevant selection procedure or concatenate latent variables extracted from different statistical methods. Herein, the mid-level metabonomics fusion of NIR, HPLC and MS data was investigated. PCA was respectively conducted with various combinations of these techniques, specifically NIR-HPLC, HPLC-MS, NIR-MS and NIR-HPLC-MS (Table 2).

Compared to the primary metabonomics analyses for HPLC and NIR alone, the principal factor total cumulative based on the results of the fusion of NIR and HPLC data was higher, with a value of $93.5 \%$. However, the discrimination was still 
Table 1 Primary data analysis results for the three techniques ${ }^{a}$

\begin{tabular}{|c|c|c|c|c|c|c|c|c|}
\hline Technique & \multicolumn{2}{|c|}{ PCA } & \multicolumn{6}{|l|}{ PLS } \\
\hline NIR & 5 & $39.0 \%$ & 3 & $97.2 \%$ & $55.2 \%$ & 0.504 & 0.504 & 0.037 \\
\hline LC-MS & 6 & $72.0 \%$ & 2 & $94.9 \%$ & $82.9 \%$ & -0.264 & 0.439 & $3.73 \times 10^{-11}$ \\
\hline
\end{tabular}

${ }^{a}$ Lvs: the number of latent variables.

unsatisfactory. NSF and SF samples were dispersed in the threedimensional space, indicating that the key information about the discrimination between the two analyses might not be captured (Fig. 2A). HPLC-MS-, NIR-MS- and NIR-HPLC-MSbased metabonomics data fusion generated summarized principal factorial plane results that were similar to the result for MS analysis mentioned above. Besides, many more variables were in the MS data set than in the HPLC data set, and hence the information contained in the MS data set could cover up the limited information of HPLC to some extent. Therefore, the results from MS-HPLC metabonomics data fusion were similar to those from primary metabonomics based on the MS data set (Table 3).

3.3.2 Mid-level metabonomics data fusion analysis with variable selection. Neither primary data analysis nor data fusion of different analytical technologies without variables selection could distinctly discriminate SF from NSF samples. This observation indicated that data fusion could not increase the classification capability, as the obtained results from metabonomics data fusion analysis were not improved greatly

Table 2 The results of the mid-level data fusion analysis for the three techniques

\begin{tabular}{llllll}
\hline & \multicolumn{2}{l}{ PCA } & & \multicolumn{2}{l}{ PLS-PCA } \\
\cline { 2 - 3 } \cline { 5 - 6 } Techniques & Lvs & $R^{2}(X)$ & & Lvs & $R^{2}(X)$ \\
\hline NIR-HPLC & 5 & $93.5 \%$ & & 5 & $55.2 \%$ \\
HPLC-MS & 5 & $71.4 \%$ & 6 & $79.4 \%$ \\
NIR-MS & 6 & $75.0 \%$ & & 5 & $66.9 \%$ \\
NIR-HPLC-MS & 6 & $74.4 \%$ & 5 & $66.7 \%$
\end{tabular}

A

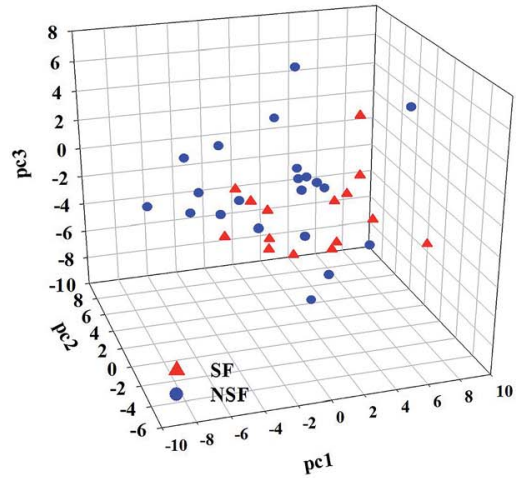

D

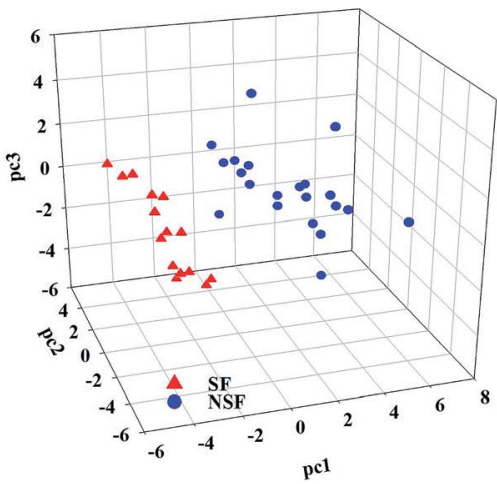

B



$\mathbf{E}$

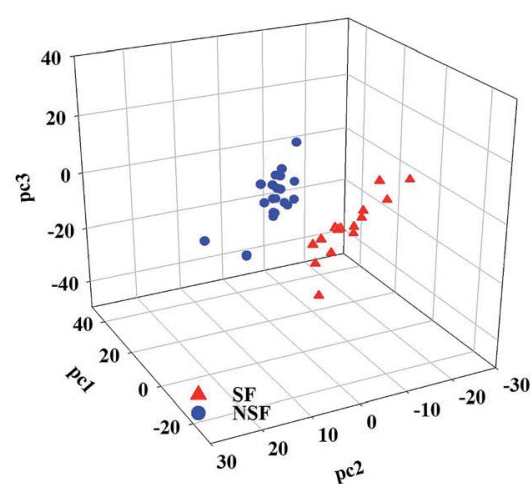

C

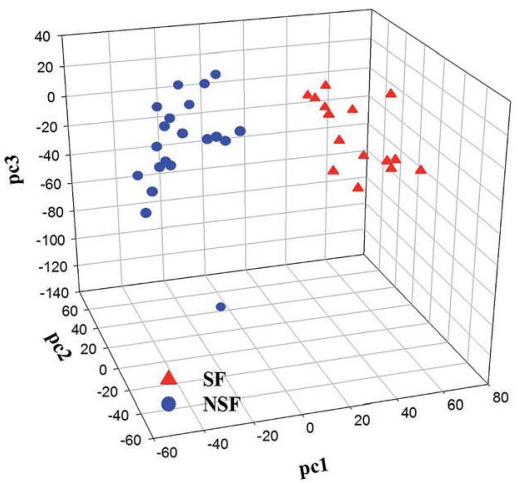

F

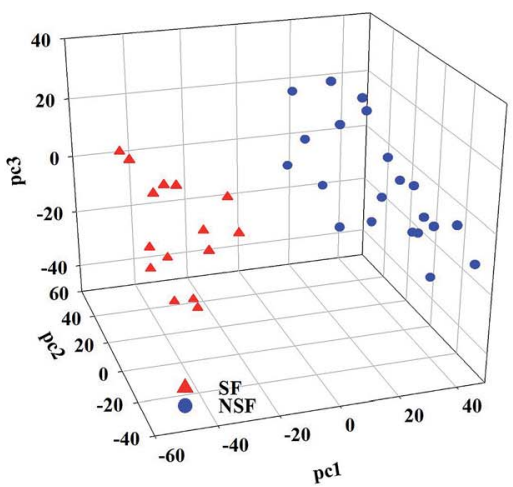

Fig. 1 The results of primary metabonomics data fusion analysis. (A-C) PCA for HPLC-DAD, NIR and LC-MS; (D-F) PLS-DA for HPLC-DAD, NIR and LC-MS. 
HPLC-NIR

PCA datafusion

(A)

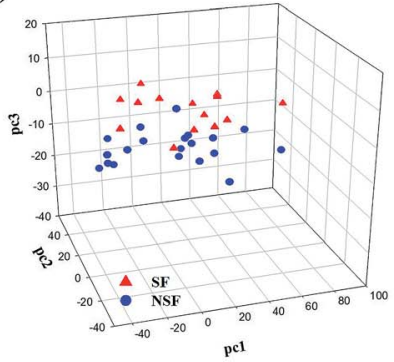

PLS-DA datafusion

(E)

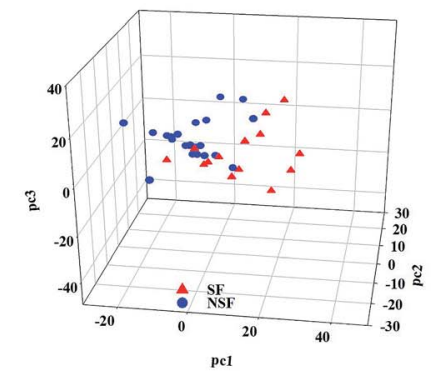

HPLC-MS

(B)

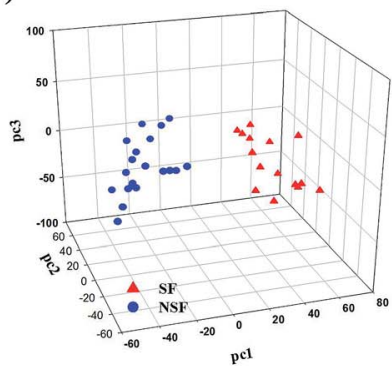

(F)

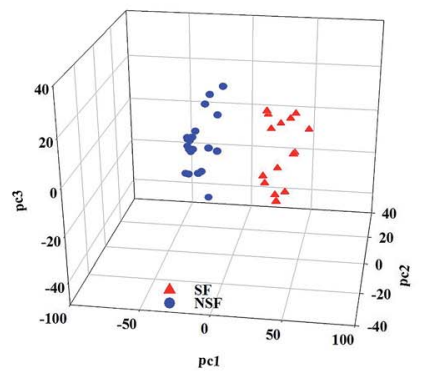

NIR-MS

(C)



(G)

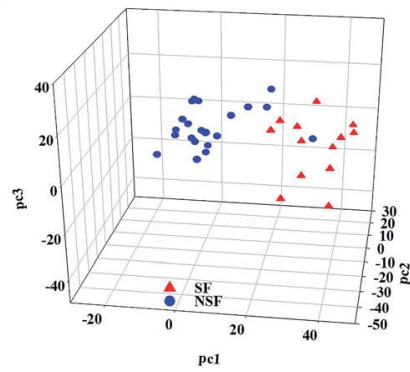

NIR-HPLC-MS

(D)

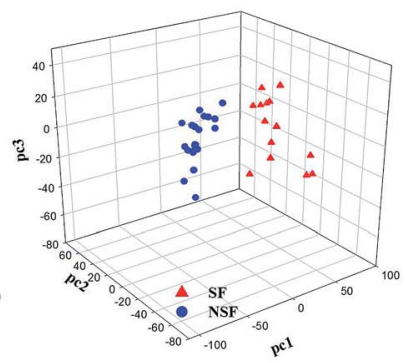

(H)

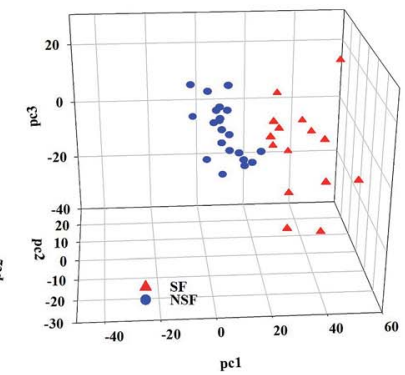

Fig. 2 The results of mid-level metabonomics data fusion analysis. (A-D) Mid-level metabonomics data fusion analysis without variable selection for NIR-HPLC, HPLC-MS, NIR-MS and NIR-HPLC-MS. (E-H) Mid-level metabonomics data fusion analysis with variable selection for NIR-HPLC, HPLC-MS, NIR-MS and NIR-HPLC-MS.

when compared to those obtained from the primary data analysis.

Subsequently, we investigated the mid-level metabonomics data fusion with PLS-DA to improve the group separation. The results from NIR-HPLC, HPLC-MS, NIR-MS and NIR-HPLC-MS data fusions were respectively presented after preliminarily screening all of data acquired from an individual platform according to variable importance values (VIP $>1.0$ ). The initial NIR, HPLC and MS data sets included 1557, 90, and 5000 variables, respectively. After the screening based on the VIP scores, 607, 27 and 1843 variables, respectively, were considered to be the most effective variables and hence retained for the subsequent discrimination. So now, a new PCA model could be constructed to enhance the group discrimination of SF and NSF samples based on the generated variables data set.

HPLC-NIR data fusion using the new PCA model yielded much better results than ever before (Fig. 2E), even though 37 batches of FLJ were not distinctly clustered into two groups. Meanwhile, the HPLC-MS data fusion generated much better results without any misclassification, while one misclassification was still found in the NIR-MS data fusion results (no. 16 was still far from the NSF group) (Fig. 2F and G).

Fig. $2 \mathrm{H}$ shows the results obtained from NIR-MS-HPLC metabonomics data fusion. Although no. 16 was not correctly allocated into the NSF group, the group discrimination potential was significantly improved when compared with those obtained from metabonomics data fusion without variable selection. Thus it could be seen from the results that metabonomics data fusion with variable selection made greater improvements in class separation than did the metabonomics data fusion without variable selection.

3.3.3 Identification of the markers discriminating NSF and SF FLJ samples based on LC-MS metabonomics analysis. As LCMS could make up for the drawbacks in the structural identification of the discriminatory markers, a UHPLC-LTQ-Orbitrap high-resolution mass spectrometer was employed to perform the discrimination of the SF and NSF FLJ samples. To obtain satisfactory group separation based on differential variables and precisely distinguish the discriminatory markers, we applied the VIP values to filter several variables that contributed to them. Meanwhile, $S$-plots and $t$-tests were also typically used for identification of the discriminatory markers and selection of the informative correlations between the markers and the modeled classes. Therefore, the filtered markers required certain conditions to be satisfied, specifically position in $S$ plot $\left(|p|>0.05\right.$ and $\left.\left|p_{\text {corr }}\right|>0.3\right)$, VIP value $(>1.0)$ and $t$-test $(p<0.05)$ (Fig. 3E). As a result, the number of original variables was 5000 . Then, 76 peaks were chosen as potential markers. Because the peak areas of most screened discriminatory variables were too low to obtain their $\mathrm{MS}^{n}$ data, a parent ion list-dynamic exclusion (PIL-DE)-based method for acquiring data was utilized to accomplish the comprehensive acquisition of HRMS ${ }^{1}$ and $\mathrm{MS}^{n}$ data sets, which greatly helped in the following structural identification. $^{36}$

Markers 1, 2 and 3 yielded identical $[\mathrm{M}-\mathrm{H}]^{-}$ions at an $\mathrm{m} / \mathrm{z}$ value of $353.0867\left(\mathrm{C}_{16} \mathrm{H}_{23} \mathrm{O}_{10}\right.$, mass error within $\left.\pm 5 \mathrm{ppm}\right)$ in 
Table 3 Identification of discriminatory markers in SF and NSF FLJ using UHPLC-LTQ-Orbitrap MS

\begin{tabular}{|c|c|c|c|c|c|}
\hline No. & $t_{\mathrm{R}}$ & $\begin{array}{l}\text { Experimental } \\
\text { mass }\end{array}$ & Formula $[\mathrm{M}-\mathrm{H}]^{-}$ & MS/MS fragment ions & Identification \\
\hline $\mathrm{M}^{a}$ & 4.47 & 353.0869 & $\mathrm{C}_{16} \mathrm{H}_{17} \mathrm{O}_{9}$ & $\operatorname{MS}^{2}[353]: 191,179,135$ & 3-CQA \\
\hline $\mathbf{M} 2^{a}$ & 6.91 & 353.0858 & $\mathrm{C}_{16} \mathrm{H}_{17} \mathrm{O}_{9}$ & $\operatorname{MS}^{2}[353]: 191,179,161$ & 5-CQA \\
\hline $\mathrm{M}^{a}{ }^{a}$ & 7.73 & 353.0856 & $\mathrm{C}_{16} \mathrm{H}_{17} \mathrm{O}_{9}$ & $\operatorname{MS}^{2}[353]: 173,179,191,135$ & 4-CQA \\
\hline $\mathrm{M} 4^{a}$ & 2.14 & 373.1122 & $\mathrm{C}_{16} \mathrm{H}_{21} \mathrm{O}_{10}$ & $\operatorname{MS}^{2}[373]: 193,149,167,179,119$ & Swertiamarin \\
\hline $\mathrm{M}^{a}{ }^{a}$ & 5.30 & 373.1118 & $\mathrm{C}_{16} \mathrm{H}_{21} \mathrm{O}_{10}$ & $\operatorname{MS}^{2}[373]: 211,167,149,193,179$ & Secologanic acid \\
\hline M6 & 7.88 & 373.1118 & $\mathrm{C}_{16} \mathrm{H}_{21} \mathrm{O}_{10}$ & $\operatorname{MS}^{2}[373]: 193,149,167,179$ & Swertiamarin isomer \\
\hline M7 & 4.23 & 375.1292 & $\mathrm{C}_{16} \mathrm{H}_{23} \mathrm{O}_{10}$ & $\operatorname{MS}^{2}[375]: 213,169,151$ & Loganin acid isomer \\
\hline M8 & 4.84 & 375.1280 & $\mathrm{C}_{16} \mathrm{H}_{23} \mathrm{O}_{10}$ & $\operatorname{MS}^{2}[375]: 213,169,151,195$ & Loganin acid \\
\hline M9 & 5.84 & 375.1273 & $\mathrm{C}_{16} \mathrm{H}_{23} \mathrm{O}_{10}$ & $\operatorname{MS}^{2}[375]: 213,169,151$ & Loganin acid isomer \\
\hline M10 & 6.63 & 375.1292 & $\mathrm{C}_{16} \mathrm{H}_{23} \mathrm{O}_{10}$ & $\operatorname{MS}^{2}[375]: 195,151$ & Loganin acid isomer \\
\hline M11 & 1.81 & 391.1231 & $\mathrm{C}_{16} \mathrm{H}_{23} \mathrm{O}_{11}$ & $\operatorname{MS}^{2}[391]: 229,211,193,185,167,149$ & Secologanic acid hydrate \\
\hline M12 & 2.45 & 391.1255 & $\mathrm{C}_{16} \mathrm{H}_{23} \mathrm{O}_{11}$ & $\operatorname{MS}^{2}[391]: 211,229,193,167,149,185$ & Secologanic acid hydrate \\
\hline M13 & 14.33 & 403.1223 & $\mathrm{C}_{17} \mathrm{H}_{23} \mathrm{O}_{11}$ & $\operatorname{MS}^{2}[403]: 371,223,179,121,91$ & Secologanin \\
\hline M14 & 1.63 & 433.0428 & $\mathrm{C}_{16} \mathrm{H}_{17} \mathrm{O}_{12} \mathrm{~S}$ & $\operatorname{MS}^{2}[433]: 241,415,353,161,191,287$ & CQA sulfate \\
\hline M15 & 2.53 & 433.0427 & $\mathrm{C}_{16} \mathrm{H}_{17} \mathrm{O}_{12} \mathrm{~S}$ & $\operatorname{MS}^{2}[433]: 415,387,353,241,353$ & CQA sulfate \\
\hline M16 & 2.66 & 433.0433 & $\mathrm{C}_{16} \mathrm{H}_{17} \mathrm{O}_{12} \mathrm{~S}$ & $\operatorname{MS}^{2}[433]: 241,415,387,259,353$ & CQA sulfate \\
\hline M17 & 4.62 & 433.0423 & $\mathrm{C}_{16} \mathrm{H}_{17} \mathrm{O}_{12} \mathrm{~S}$ & $\operatorname{MS}^{2}[433]: 415.387,259$ & CQA sulfate \\
\hline M18 & 5.01 & 433.0419 & $\mathrm{C}_{16} \mathrm{H}_{17} \mathrm{O}_{12} \mathrm{~S}$ & $\operatorname{MS}^{2}[433]: 415,241,161,259,387$ & CQA sulfate \\
\hline M19 & 1.12 & 435.0591 & $\mathrm{C}_{16} \mathrm{H}_{17} \mathrm{O}_{12} \mathrm{~S}$ & $\operatorname{MS}^{2}[435]: 353,191,179$ & CQA sulfite \\
\hline M20 & 3.15 & 437.0720 & $\mathrm{C}_{16} \mathrm{H}_{21} \mathrm{O}_{12} \mathrm{~S}$ & $\operatorname{MS}^{2}[437]: 193,149,373,355$ & Secologanic acid sulfite \\
\hline $\mathrm{M}_{21}{ }^{a}$ & 19.06 & 447.0916 & $\mathrm{C}_{21} \mathrm{H}_{19} \mathrm{O}_{11}$ & $\operatorname{MS}^{2}[447]: 285$ & Luteolin-7-O-glucoside \\
\hline M22 & 21.06 & 447.0918 & $\mathrm{C}_{21} \mathrm{H}_{19} \mathrm{O}_{11}$ & $\operatorname{MS}^{2}[447]: 285$ & Luteolin-7-O-glucoside isomer \\
\hline M23 & 1.93 & 455.0822 & $\mathrm{C}_{16} \mathrm{H}_{23} \mathrm{O}_{13} \mathrm{~S}$ & $\mathrm{MS}^{2}[455]: 373,411,437,193,211$ & Secologanic acid sulfite \\
\hline M24 & 2.15 & 455.0836 & $\mathrm{C}_{16} \mathrm{H}_{23} \mathrm{O}_{13} \mathrm{~S}$ & $\operatorname{MS}^{2}[455]: 373,437,411,193,211$ & Secologanic acid sulfite \\
\hline M25 & 18.22 & 463.0854 & $\mathrm{C}_{21} \mathrm{H}_{19} \mathrm{O}_{12}$ & $\operatorname{MS}^{2}[463]: 301,271,445$ & Hyperoside isomer \\
\hline M26 & 18.73 & 463.0861 & $\mathrm{C}_{21} \mathrm{H}_{19} \mathrm{O}_{12}$ & $\operatorname{MS}^{2}[463]: 301,445,271$ & Hyperoside \\
\hline M27 & 23.05 & 499.1231 & $\mathrm{C}_{25} \mathrm{H}_{23} \mathrm{O}_{11}$ & $\operatorname{MS}^{2}[499]: 337,173,335,353$ & 4- $p$ Co-1-CQA \\
\hline M28 & 23.49 & 499.1233 & $\mathrm{C}_{25} \mathrm{H}_{23} \mathrm{O}_{11}$ & $\operatorname{MS}^{2}[499]: 353,337,191,335,179$ & 5- $p$ Co-3-CQA \\
\hline M29 & 25.21 & 499.1230 & $\mathrm{C}_{25} \mathrm{H}_{23} \mathrm{O}_{11}$ & $\operatorname{MS}^{2}[499]: 353,337,179,191$ & 3-p Co-4-CQA \\
\hline $\mathrm{M} 30^{a}$ & 20.36 & 515.1155 & $\mathrm{C}_{25} \mathrm{H}_{23} \mathrm{O}_{11}$ & $\operatorname{MS}^{2}[515]: 353,335,173,179$ & 3,4-DiCQA \\
\hline $\mathrm{M} 1^{a}$ & 20.85 & 515.1155 & $\mathrm{C}_{25} \mathrm{H}_{23} \mathrm{O}_{11}$ & $\operatorname{MS}^{2}[515]: 353,191,179,335$ & 3,5-DiCQA \\
\hline $\mathrm{M} 32^{a}$ & 22.44 & 515.1163 & $\mathrm{C}_{25} \mathrm{H}_{23} \mathrm{O}_{11}$ & $\operatorname{MS}^{2}[515]: 353,191,179,335,353$ & 4,5-DiCQA \\
\hline M33 & 17.34 & 527.0494 & $\mathrm{C}_{21} \mathrm{H}_{19} \mathrm{O}_{14} \mathrm{~S}$ & $\operatorname{MS}^{2}[527]: 447,285,481$ & Luteolin-7-O-glucoside sulfate \\
\hline M34 & 23.82 & 529.1343 & $\mathrm{C}_{26} \mathrm{H}_{25} \mathrm{O}_{12}$ & $\operatorname{MS}^{2}[529]: 367,179,335,353,193$ & 3-C-4-FQA \\
\hline M35 & 24.60 & 529.1340 & $\mathrm{C}_{26} \mathrm{H}_{25} \mathrm{O}_{12}$ & $\operatorname{MS}^{2}[529]: 353,367,191,179$ & 5-C-3-FQA \\
\hline M36 & 25.86 & 529.1335 & $\mathrm{C}_{26} \mathrm{H}_{25} \mathrm{O}_{12}$ & $\operatorname{MS}^{2}[529]: 353,367,173,335$ & cis-5-C-3-FQA \\
\hline M37 & 8.73 & 543.0431 & $\mathrm{C}_{21} \mathrm{H}_{19} \mathrm{O}_{15} \mathrm{~S}$ & $\operatorname{MS}^{2}[543]: 463,381,525,301$ & Hyperoside sulfate \\
\hline M38 & 12.76 & 543.0432 & $\mathrm{C}_{21} \mathrm{H}_{19} \mathrm{O}_{15} \mathrm{~S}$ & $\operatorname{MS}^{2}[543]: 381,301,381,463$ & Hyperoside sulfate \\
\hline M39 & 18.80 & 593.1488 & $\mathrm{C}_{27} \mathrm{H}_{29} \mathrm{O}_{15}$ & $\operatorname{MS}^{2}[593]: 285,447$ & Lonicerin isomer \\
\hline M40 & 19.71 & 593.1483 & $\mathrm{C}_{27} \mathrm{H}_{29} \mathrm{O}_{15}$ & $\operatorname{MS}^{2}[593]: 285,447$ & Lonicerin isomer \\
\hline $\mathrm{M}_{4} 1^{a}$ & 20.50 & 593.1486 & $\mathrm{C}_{27} \mathrm{H}_{29} \mathrm{O}_{15}$ & $\operatorname{MS}^{2}[593]: 285$ & Lonicerin \\
\hline M42 & 16.70 & 595.0737 & $\mathrm{C}_{25} \mathrm{H}_{23} \mathrm{O}_{15} \mathrm{~S}$ & $\mathrm{MS}^{2}[595]: 549,577,415,241,259$ & DiCQA sulfate \\
\hline M43 & 16.98 & 595.0750 & $\mathrm{C}_{25} \mathrm{H}_{23} \mathrm{O}_{15} \mathrm{~S}$ & $\operatorname{MS}^{2}[595]: 549,577,415,301,397$ & DiCQA sulfate \\
\hline M44 & 17.61 & 595.0748 & $\mathrm{C}_{25} \mathrm{H}_{23} \mathrm{O}_{15} \mathrm{~S}$ & $\operatorname{MS}^{2}[595]: 577,549,415,433,241,259$ & DiCQA sulfate \\
\hline M45 & 17.89 & 595.0737 & $\mathrm{C}_{25} \mathrm{H}_{23} \mathrm{O}_{15} \mathrm{~S}$ & $\mathrm{MS}^{2}[595]: 577,549,415,433,241,259$ & DiCQA sulfate \\
\hline M46 & 19.38 & 595.0745 & $\mathrm{C}_{25} \mathrm{H}_{23} \mathrm{O}_{15} \mathrm{~S}$ & $\operatorname{MS}^{2}[595]: 577,549,415,433,259$ & DiCQA sulfate \\
\hline M47 & 21.25 & 595.0745 & $\mathrm{C}_{25} \mathrm{H}_{23} \mathrm{O}_{15} \mathrm{~S}$ & $\operatorname{MS}^{2}[595]: 577,415,549,433,259,241$ & DiCQA sulfate \\
\hline M48 & 22.70 & 607.1653 & $\mathrm{C}_{28} \mathrm{H}_{31} \mathrm{O}_{15}$ & $\mathrm{MS}^{2}[607]: 299$ & Chrysoeriol-7-O- $\beta$-D-neohesperidoside \\
\hline M49 & 18.30 & 609.1403 & $\mathrm{C}_{27} \mathrm{H}_{29} \mathrm{O}_{16}$ & $\mathrm{MS}^{2}[609]: 301,300,271,255,179,591$ & Rutin \\
\hline
\end{tabular}

${ }^{a}$ Identified by comparison with reference standards; CQA, caffeoylquinic acid; DiCQA, dicaffeoylquinic acid; $p$ CoCQA, $p$-coumaroylcaffeoylquinic acid; CFQA, caffeoylferuloylquinic acid.

negative ion mode. Their deprotonated molecular ions all generated a series of diagnostic fragment ions including those

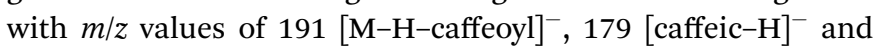
$173\left[\mathrm{M}-\mathrm{H}-\text { caffeoyl- } \mathrm{H}_{2} \mathrm{O}\right]^{-}{ }^{-37} \mathrm{CQAs}$ attributed to three different linkage positions of caffeoyl groups on quinic acid have been reported to display different intensities of their ESI-MS ${ }^{2}$ base peak ions and predominant product ions. Meanwhile, based on retention times and $\mathrm{MS}^{n}$ spectra of the corresponding reference substances and literature data, markers 1-3 were identified to be 5-CQA (Fig. S5 $†$ ), 3-CQA and 4-CQA, respectively.

Markers 14-18 generated their deprotonated $[\mathrm{M}-\mathrm{H}]^{-}$ molecular ions each at an $m / z$ of $433.0435\left(\mathrm{C}_{16} \mathrm{H}_{17} \mathrm{O}_{12} \mathrm{~S}\right.$, mass error within $\pm 5 \mathrm{ppm})$. In their ESI-MS ${ }^{2}$ spectra, the diagnostic product ions were at $m / z$ values of $415\left[\mathrm{M}-\mathrm{H}-\mathrm{H}_{2} \mathrm{O}\right]^{-}, 387[\mathrm{M}-\mathrm{H}-$ 
A

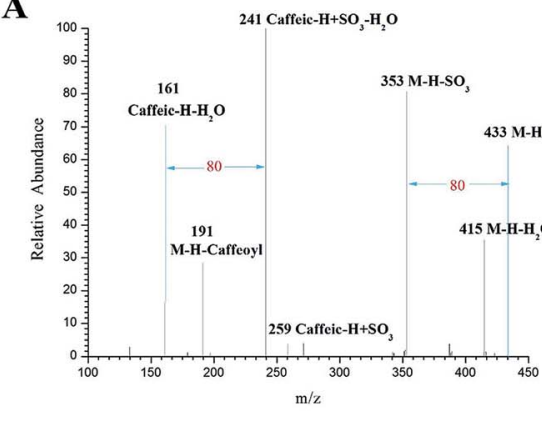

C

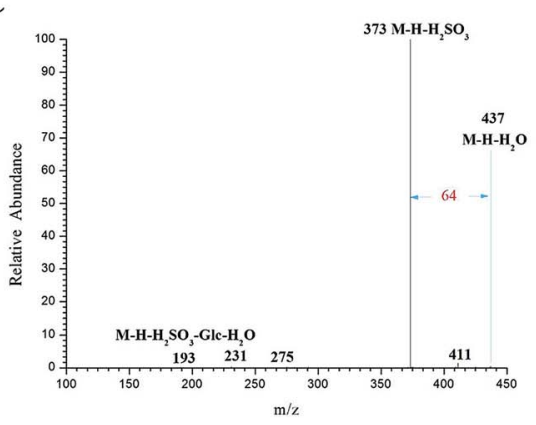

$\mathbf{E}$



B

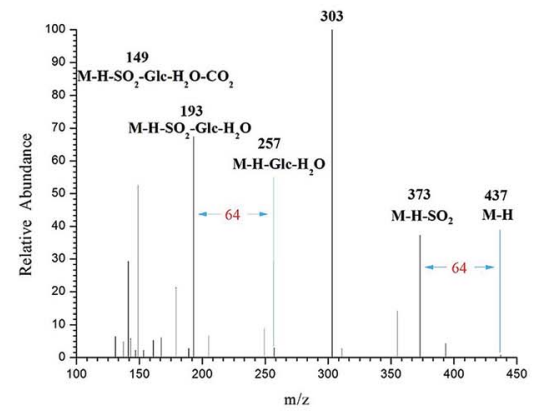

D

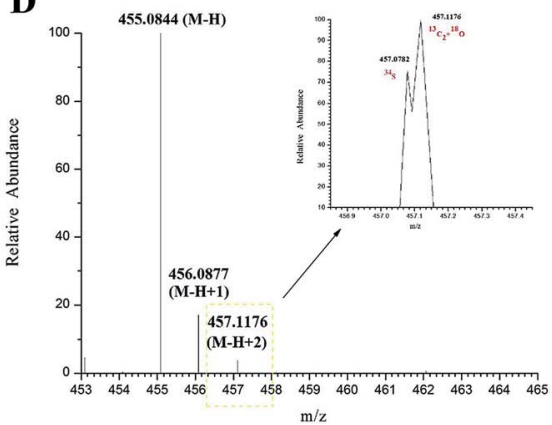

Fig. 3 The mass fragmentation behaviors of identified markers. (A) HRMS ${ }^{1}$ spectrum of M14. (B) ESI-MS ${ }^{2}$ spectrum of M20. (C) HRMS ${ }^{1}$ spectrum of M23. (D) ESI-MS ${ }^{2}$ spectrum of M24. (E) The S-plot of LC-MS metabonomics analysis.

$\left.\mathrm{H}_{2} \mathrm{O}-\mathrm{CO}\right]^{-}, 353\left[\mathrm{M}-\mathrm{H}-\mathrm{SO}_{3}\right]^{-}, 259\left[\text { caffeic- } \mathrm{H}+\mathrm{SO}_{3}\right]^{-}$and 241 [caffeic- $\left.\mathrm{H}+\mathrm{SO}_{3}-\mathrm{H}_{2} \mathrm{O}\right]^{-}$. The observation of the pair of ions at $m /$ $z$ values of 433 and 353 (Fig. 3A) further confirmed that the sulfate moiety was introduced to the CQA molecule, which has to the best of our knowledge never been reported before. Finally, markers $14-18$ were tentatively identified as isomeric CQA sulfate.

Similarly, the ESI-MS ${ }^{2}$ spectrum of marker 20 (Fig. 3B) showed an $\mathrm{m} / \mathrm{z}$ signal corresponding to its deprotonated $[\mathrm{M}-\mathrm{H}]^{-}$ molecular ion at a value of $437.0748\left(\mathrm{C}_{16} \mathrm{H}_{21} \mathrm{O}_{12} \mathrm{~S}\right.$, error within $\pm 5 \mathrm{ppm})$. Moreover, the characteristic product ions at $\mathrm{m} / \mathrm{z}$ values of $373\left[\mathrm{M}-\mathrm{H}-\mathrm{SO}_{2}\right]^{-}, 193\left[\mathrm{M}-\mathrm{H}-\mathrm{SO}_{2}-\mathrm{Glc}_{-} \mathrm{H}_{2} \mathrm{O}\right]^{-}$and 149 $\left[\mathrm{M}-\mathrm{H}-\mathrm{SO}_{2}-\mathrm{Glc}-\mathrm{H}_{2} \mathrm{O}-\mathrm{CO}_{2}\right]^{-}$were all observed. Based on the observation of the signals at the $\mathrm{m} / \mathrm{z}$ values of 193 and 149 coupled with its $[\mathrm{M}-\mathrm{H}]^{-}$ion, marker 20 may be concluded to be secologanic acid. ${ }^{38}$ Meanwhile, the observation of the product ion at the $\mathrm{m} / \mathrm{z}$ value of 373 confirmed that the sulfite moiety was introduced into the iridoid molecule. Accordingly, marker 20 was tentatively identified as isomeric secologanic acid sulfate.

In addition, a combination of the isotopic pattern combined and chromatography analyses was used for screening sulfurcontaining compounds in the complex systems, mainly because the ${ }^{34} \mathrm{~S}$ isotopic ion has been shown to be drastically affected by ${ }^{13} \mathrm{C}_{2}$ and ${ }^{18} \mathrm{O} .{ }^{39}$ Markers 23 and 24 produced their $[\mathrm{M}-\mathrm{H}]^{-}$ions each at an $\mathrm{m} / z$ of $455.0822\left(\mathrm{C}_{16} \mathrm{H}_{23} \mathrm{O}_{12} \mathrm{~S}\right.$, error within $\left.\pm 5 \mathrm{ppm}\right)$. And both of them generated a series of fragment ions at $\mathrm{m} / \mathrm{z}$ values of $437\left[\mathrm{M}-\mathrm{H}-\mathrm{H}_{2} \mathrm{O}\right]^{-}, 411\left[\mathrm{M}-\mathrm{H}-\mathrm{CO}_{2}\right]^{-}, 373[\mathrm{M}-\mathrm{H}-$ $\left.\mathrm{H}_{2} \mathrm{SO}_{3}\right]^{-}, 211\left[\mathrm{M}-\mathrm{H}-\mathrm{H}_{2} \mathrm{SO}_{3}-\mathrm{Glc}\right]^{-}$and $193\left[\mathrm{M}-\mathrm{H}-\mathrm{H}_{2} \mathrm{SO}_{3}-\mathrm{Glc}-\right.$ $\left.\mathrm{H}_{2} \mathrm{O}\right]^{-}$. Furthermore, they simultaneously produced the isotopic patterns of the ${ }^{34} \mathrm{~S}$ ion at an $\mathrm{m} / z$ of 457.07822 and of the ${ }^{13} \mathrm{C}_{2}+{ }^{18} \mathrm{O}$ ion at an $\mathrm{m} / \mathrm{z}$ of 457.11760 . Their characteristic product ions at $\mathrm{m} /$ $z$ values of 437 and 373 probably resulted from the occurrence of the sulfite moiety in some of the iridoid molecules. Accordingly, markers 23 and 24 were putatively identified as secologanic acidsulfite or its isomers (Fig. 3C and D).

Taken together, a total of 49 discriminatory markers (Table $\mathrm{S} 4 \dagger)$ attributed to iridoids, organic acids and flavones were screened and characterized according to the fragmentation behaviors, isotopic patterns and diagnostic product ions obtained using the UHPLC-LTQ-Orbitrap MS coupled with the established integrated strategy. Eighteen of these markers were assigned to sulfate/sulfite derivatives of iridoid and chlorogenic acid, which could be chosen as the characteristic Q-markers for SF FLJ discrimination. ${ }^{40}$ (Note that Fig. S6† shows a histogram of signal intensities of sulfur derivatives.)

3.3.4 Multi-omics correlation analysis (MOCA). At first, we performed a selection of specific wavenumbers according to the NIR-based metabonomics data analysis. Analysis of the available NIR spectra, specifically of the different NIR wavebands, quickly provided vast amounts of useful chemical information. However, it was in fact unable to present selective valid wavebands with discriminating potential. In order to interpret the sulfur fumigation process and screen the potential wavebands that presented the significant differences between SF and NSF samples, synergy interval partial least squares (siPLS) analysis with three intervals was employed to obtain the significant differences between SF and NSF samples. To eliminate the influence of overfitting, we set the latent variables to be within the range 1-10. As demonstrated in Table 4, the siPLS analysis with SG11 $+2^{\text {nd }}$ optimized (the other siPLS methods are shown 
Table 4 The results of SiPLS analysis

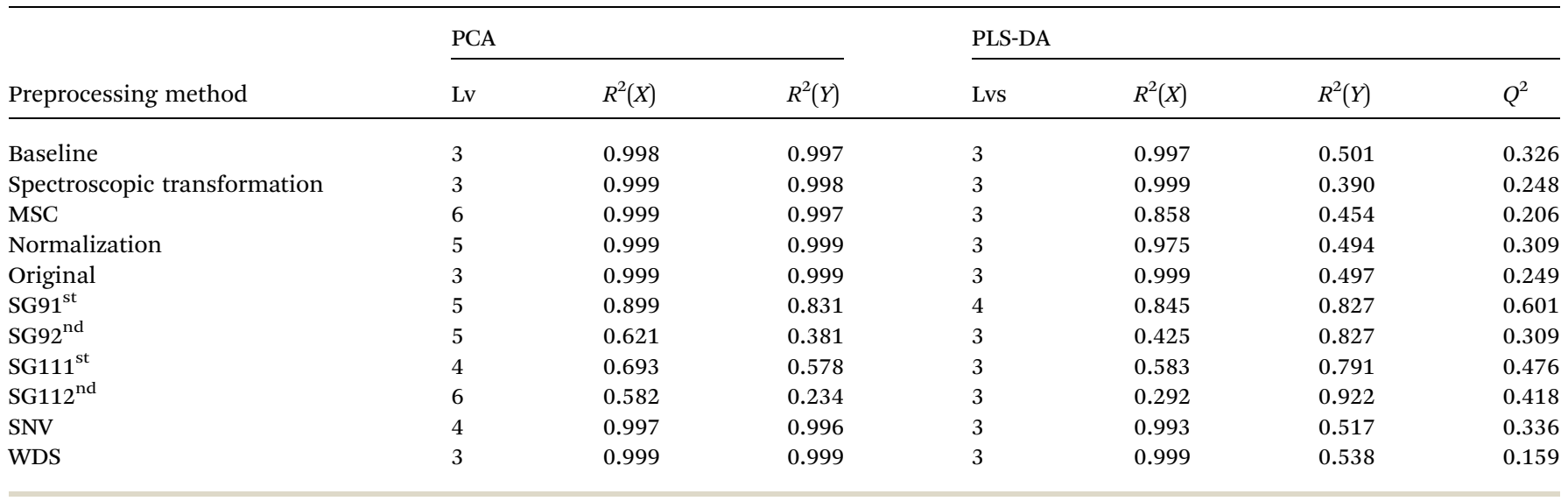

in Fig. S7 and S8 $\dagger$ ) was the best discriminatory model and achieved a value of 0.859 for $R^{2}(Y)$ and 0.471 for $Q^{2}$; i.e., this method produced a better model performance than did any of the other preprocessing methods. Furthermore, the SF and NSF FLJ samples were clearly separated (Fig. 4A). The wavenumbers of the optical subinterval combinations ranged from 5000 to $5200 \mathrm{~cm}^{-1}$. Thereafter, NIR spectra were integrated with twodimensional correlation spectra (2D-COS) that were expected to clearly identify the screened wavebands, discriminate features and details of the structural changes of SF and NSF FLJ samples. For the synchronous 2D-COS auto-peak analysis, two main sensitive variables were identified and displayed wavenumbers ranging from 5000 to $5200 \mathrm{~cm}^{-1}$ (see Fig. 4B, which shows the diagonal data of the 2D-COS plot, and Fig. S9, $\dagger$ which shows the 2D-COS plot), whose correlation analysis was in accordance with the wavebands screened using the siPLS method. Similar to that previously reported, strong absorptions in the wavenumber range $5000-5200 \mathrm{~cm}^{-1}$ were attributed to $\mathrm{S}-\mathrm{H}$ and $\mathrm{S}-\mathrm{OH} .{ }^{33,41}$ As mentioned above, some sulfur-containing components were identified as the discriminatory markers. As a result, a close relationship was identified between the screened wavebands $\left(5000-5200 \mathrm{~cm}^{-1}\right)$ and the sulfurcontaining markers generated in the sulfur fumigation process.

To validate the above-mentioned results, one of the main representative chemical constituents, namely chlorogenic acid (5-CQA), was subjected to sulfur fumigation and analyzed using
(A)

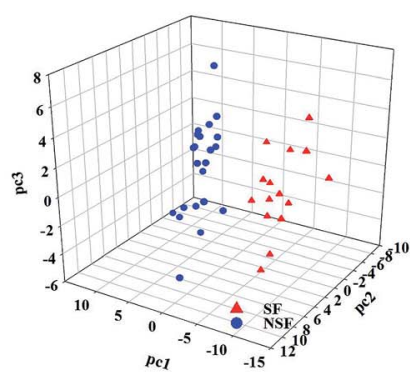

(C)

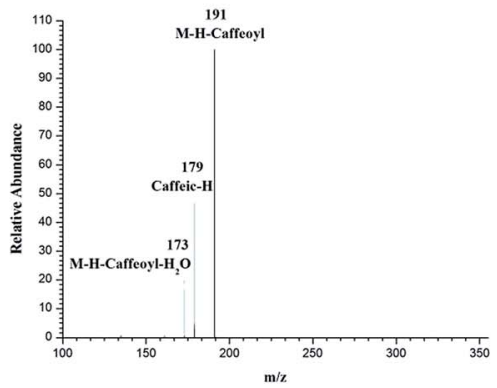

(B)

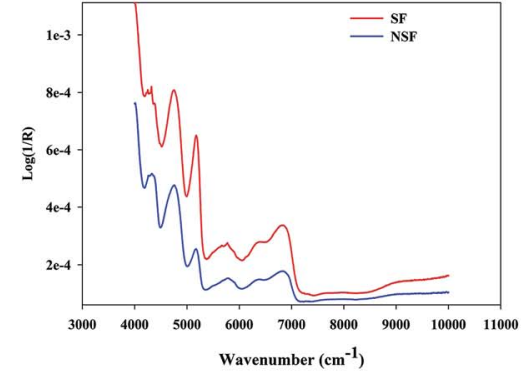

(E)



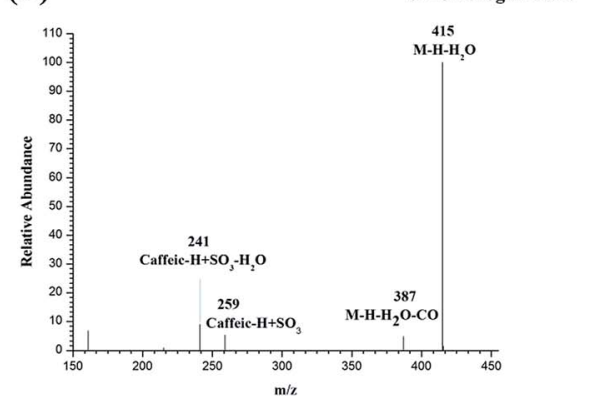

Fig. 4 The MOCA for the FLJ. (A) The discriminatory information of preprocess method of SG9+1 ${ }^{\text {st; }}$ (B) the synchronous $2 \mathrm{D}$-COS auto-peak analysis of the SF and NSF samples; (C) the mass fragmentation behaviors of SF chlorogenic acid; (D) the synchronous 2D-COS auto-peak analysis of the SF and NSF chlorogenic acid samples; (E) the mass fragmentation behaviors of SF chlorogenic acid. 
the same methods. The autocorrelation curves of the SF and NSF chlorogenic acid samples (Fig. 4D) were derived from their respective 2D-COS spectra. Obvious differences between the SF and NSF chlorogenic acid samples in the wavebands between 5000 and $5200 \mathrm{~cm}^{-1}$ were observed, which was also in accordance with the wavebands screened using the siPLS model. The subsequent LC-HRMS analysis of an SF chlorogenic acid mixture also indicated the presence of newly generated constituents (Fig. 4C and E) except for the prototype drug during the process of sulfur fumigation. Through analyzing the fragment ions of the S-derivatives, it was found that $\mathrm{SO}_{3}$ (79.9568) and $\mathrm{H}_{2} \mathrm{SO}_{3}$ (81.9725) were the characteristic neutral losses of organic sulfates or sulfites. The assignments of these newly emerged peaks were confirmed to be the rudimentary sulfate derivatives of chlorogenic acid based on the HRMS data, which indicated a mass $79.95 \mathrm{Da}\left(\mathrm{SO}_{3}\right)$ more than that of standard reference. It also indicated that the results of NIR were reliable and credible in the discrimination of SF FLJ.

\subsection{Proposed protocol for the rapid detection and} mechanistic explanation of the sulfur fumigation of CHMs using SF FLJ as a study case

Evaluating the quality of CHMs and judging their authenticity are two major challenges. Sulfur fumigation has attracted increasing attention due to its alteration of CHM quality resulting from its damage to bioactive components, generating excessive sulfur dioxide residue and especially changing the chemically active ingredients in CHMs.

NIR, HPLC and LC-MS were proposed to be used to evaluate the quality of CHMs. However, the variations during sulfur fumigation were much more complicated than expected. Furthermore, the amount of data obtained based on one single method was still limited, making it difficult to expound on the mechanism of sulfur fumigation. To experimentally support our inference, we selected FLJ as a model herb in this study. With the development of a few high-throughput strategies, integrative metabonomics analysis was applied to integrate the multiple interactions of NIR spectra, HPLC chromatograms and HRMS data. The results aimed to reveal whether the herb underwent sulfur fumigation and to illuminate the inherent mechanism of the NIR judgment method by associating NIR with HPLC and UHPLC-MS analyses. Our established approach was applied to rapidly discriminate SF FLJ among many unknown samples, and is expected to be greatly beneficial for guaranteeing CHM quality.

To perform the analysis of the sulfur fumigation of CHMs, the process of sulfur fumigation was first simulated in the laboratory. According to the characteristics of each analytical technology, optimum analytical conditions were adopted and the corresponding high-quality data of SF and NSF CHM samples were
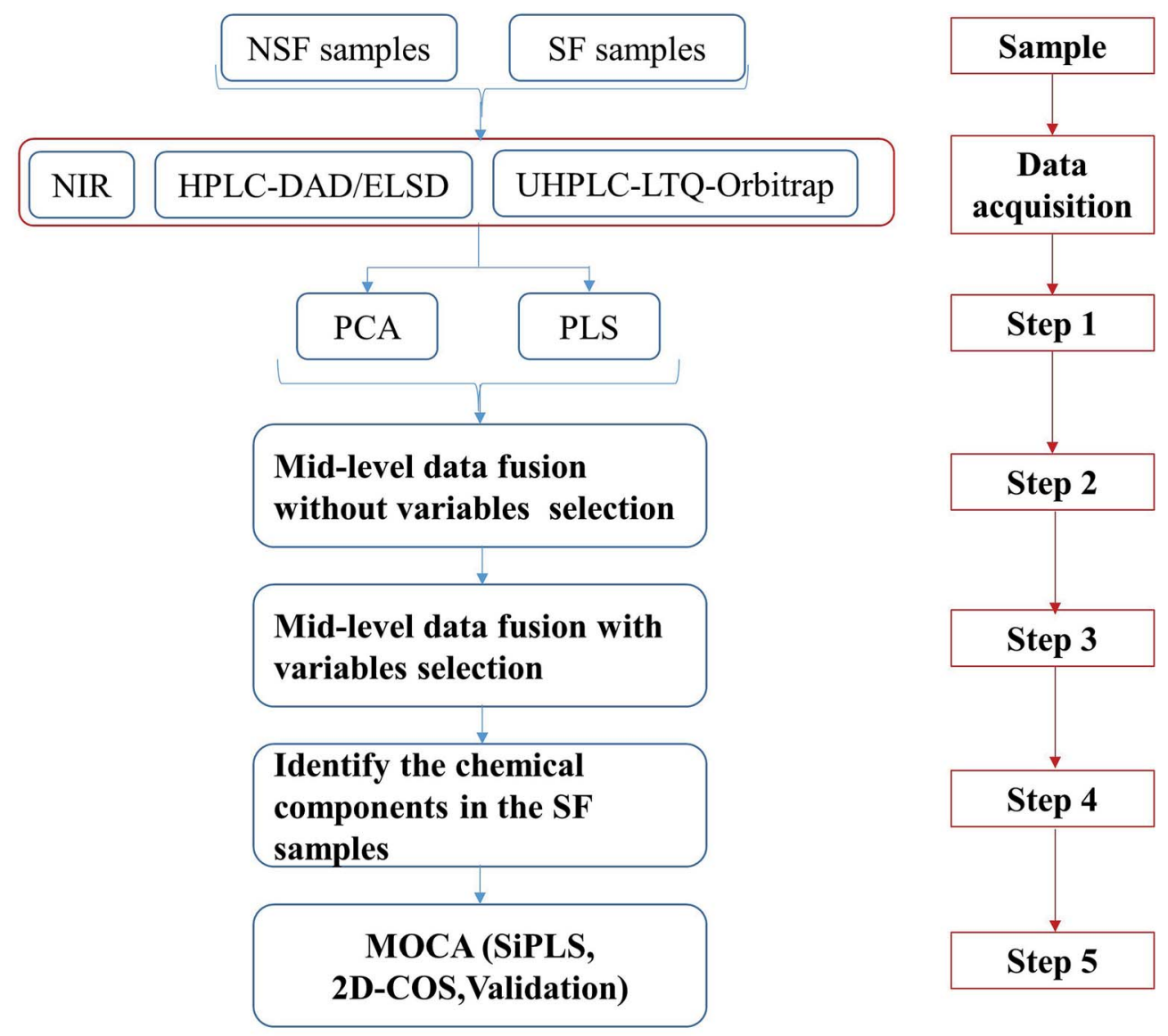

Fig. 5 A suggested protocol for the rapid discrimination of SF CHMs and an explanatory mechanism. 
obtained. All of these experiments provided the foundation for subsequent data analysis, which is illustrated in Fig. 5 .

Step 1: Performing PCA and PLS-DA for the single technology.

This step was focused on the analytical capability of each single technology and whether the SF and NSF CHMs could be distinguished. Our study demonstrated that NIR spectroscopy based on a data preprocessing method $\left(\mathrm{SG9}+2^{\text {nd }}\right)$ with a multivariate calibration approach such as PCA and PLS-DA was the appropriate tool to discriminate SF from NSF FLJ samples. The chemical constituents in FLJ samples displayed strong ultraviolet absorption, which was observed with HPLC-DAD at $330 \mathrm{~nm}$, $238 \mathrm{~nm}, 254 \mathrm{~nm}$ and $280 \mathrm{~nm}$. Peak areas ( $\geq 150 \mathrm{mAU})$ were selected separately through the data fusion of the four wavelengths and then analyzed by performing PCA and PLS-DA. In addition, a UHPLC-LTQ-Orbitrap high-resolution MS was employed to comprehensively and dynamically profile the chemical constituents in FLJ. The derivative content during the sulfur fumigation process was not abundant enough, i.e., the signals of sulfur-containing analytes would have been drowned out by the contribution of inherent constituents.

Step 2 and Step 3: Performing the integrative metabonomics analysis, such as mid-level metabonomics data fusion analysis without/with variable selection.

In our previous study, the data fusion of NIR- and HRMSbased metabonomics-like analysis was successfully applied to accomplishing the discrimination of SF Ophiopogon Radix. ${ }^{17}$ Herein, we combined three kinds of analytical techniques including NIR, HPLC and UHPLC-HRMS to obtain the dimensional information of SF samples, and investigated two types of mid-level metabonomics data fusion strategies as illustrated in Step 2 and Step 3. For that, informative features of the raw data from a single instrument were separately extracted using their own protocol from sample preparation to data preprocessing.

The comparison between the unique model and the metabonomics data fusion model is illustrated in Fig. 6. No single analytical platform could be utilized to accurately discriminate the SF samples based on PCA score plots. HPLC and NIR led to classification without rhyme or reason and HRMS could not correctly discriminate one of the SF samples (no. 16). Thus, we believed that utilizing mid-level metabonomics data fusion without variable selection to obtain more accurate characteristics of the samples might be a much better choice. As a result, the potential to discriminate between of NSF and SF samples was actually improved with no. 16 still in the wrong class, and the results were worse than the individual application of MS. Mid-level fusion with variable selection was employed and clearly improved the class separation, as samples were correctly classified and less scattered (Fig. 6K). Taking the classification into account, the fusion of NIR and HRMS data, accomplished with high accuracy, provided the best model (Fig. 6I and J). Moreover, variables that were selected before classification generated better classification results than those obtained when all variables were used. Overall, the proposed metabonomics data fusion approach demonstrated an ability to effectively discriminate key information from raw analysis data.



Fig. 6 Comparison between the unique model and the metabonomics data fusion model. (A-C) PCA for HPLC-DAD, NIR and LC-MS. (D-G) Result for HPLC-NIR, HPLC-MS, NIR-MS and NIR-HPLC-MS data fusion without variable selection analysis. (H-K) Result for HPLC-NIR, HPLCMS, NIR-MS and NIR-HPLC-MS data fusion with variable selection analysis. 
The results demonstrated that the mid-level metabonomics data fusion methods were much better than all of the primary analyses, which meant that the information obtained from individual techniques was in fact insufficient. The results from both kinds of mid-level fusion strategies accomplished the effective discrimination of SF FLJ samples.

Step 4: Identifying the discriminatory markers attributed to group separation.

LTQ-Orbitrap high-resolution MS has been one of the most powerful approaches used for the rapid identification of multiple constituents in CHMs. ${ }^{\mathbf{4 2 , 4 3}}$ It has been used to combine high trapping capacity and multiple data acquisition of linear ion traps to generate a large amount of information from $\mathrm{MS}^{1}$ and $\mathrm{MS}^{n}$ data. In this study, a highly sensitive and effective strategy was utilized for rapidly screening and identifying SF FLJ by using PIL-DE acquisition based on a hybrid LTQ-Orbitrap mass spectrometer to accomplish the overall acquisition of data sets, which helped allow for a search of a greater number of potential active compounds especially for the sulfur-containing constituents. As a result, 49 markers including iridoids, organic acids and the sulfur-containing derivatives were positively or tentatively identified.

Step 5: Application of MOCA for deriving mechanistic explanations of the sulfur fumigation process and the corresponding method validation.

An NIR spectrum was constructed from different wavebands, but not every waveband displayed a special discrimination ability. Therefore, siPLS analysis was employed to screen the potential wavebands that presented the significant differences between SF and NSF samples. In step 4 mentioned above, some sulfur-containing constituents were identified that would explain the potential NIR wavebands. Chlorogenic acid was selected as the example to validate whether the new sulfurcontaining derivatives were produced after the sulfur fumigation process.

\section{Conclusions}

Our work indicated that the proposed protocol for the rapid detection of SF CHMs is also beneficial for revealing the intrinsic mechanism of sulfur fumigation and boosting the ability to discriminate SF from NSF FLJ samples, and could serve as an example for future research on rapidly detecting other SF CHMs. Integrative metabonomics analysis was also found to be beneficial for evaluating the quality of and rapidly detecting CHMs. Our work also suggests a future trend of integrating multiple metabonomics datasets from different technologies to achieve a sound evaluation.

\section{Conflicts of interest}

All authors declare that they have no conflict of interest.

\section{Acknowledgements}

This work has been financially supported by National Natural Science Foundation of China (No. 81303206), Beijing National
Natural Science Foundation (No. 7173265) and Beijing Nova Program (Z171100001117029).

\section{References}

1 C. Kang, C. J. Lai, D. Zhao, T. Zhou, D. H. Liu, C. Lv, S. Wang, L. Kang, J. Yang and Z. L. Zhan, J. Hazard. Mater., 2017, 340, 221-230.

2 M. Kong, H. H. Liu, J. Wu, M. Q. Shen, Z. G. Wang, S. M. Duan, Y. B. Zhang, H. Zhu and S. L. Li, J. Ethnopharmacol., 2017, 212, 95-105.

3 A. L. Guo, L. M. Chen, X. Q. Liu, Q. W. Zhang, H. M. Gao, Z. M. Wang, X. Wei and Z. Z. Wang, Molecules, 2014, 19, 16640-16655.

4 W. L. T. Kan, B. Ma and G. Lin, Front. Pharmacol., 2011, 2, 84. 5 X. Jiang and L. F. Huang, Sulfur fumigation, Phytomedicine, 2013, 20, 97-105.

6 M. Q. Carter, M. H. Chapman, F. Gabler and M. T. Brandl, Food Microbiol., 2015, 49, 189-196.

7 L. Zhang, H. Shen, J. Xu, J. D. Xu, Z. L. Li, J. Wu, Y. T. Zou, L. F. Liu and S. L. Li, Food Chem., 2018, 246, 202-210.

8 S. M. Duan, J. Xu, Y. J. Bai, Y. Ding, M. Kong, H. H. Liu, X. Y. Li, Q. S. Zhang, H. B. Chen and L. F. Liu, Food Chem., 2016, 192, 119-124.

9 B. Ma, W. L. T. Kan, Z. He, S. L. Li and L. Ge, J. Ethnopharmacol., 2017, 195, 222-230.

10 R. J. Li, X. J. Kou, J. J. Tian, Z. Q. Meng, Z. W. Cai, F. Q. Cheng and C. Dong, Chemosphere, 2014, 112, 296-304.

11 A. Agar, V. Küçükatay, P. Yargicoglu, S. Bilmen, S. Gümüşlü and G. Yücel, Diabetes Metab., 2000, 26, 140-144.

12 Pharmacopoeia of People's Republic of China, Part L, China Medical Science and Technology Press, Beijing, 2005.

13 X. Jin, L. Y. Zhu, H. Shen, J. Xu, S. L. Li, X. B. Jia, H. Cai, B. C. Cai and R. Yan, Food Chem., 2012, 135, 1141-1147.

14 M. Kong, H. H. Liu, J. Xu, C. R. Wang, M. Lu, X. N. Wang, Y. B. Li and S. L. Li, J. Pharm. Biomed. Anal., 2014, 98, 424433.

15 X. Chen, X. Li, X. Mao, H. Huang, T. Wang, Z. Qu, M. Jing and W. Gao, Food Chem., 2017, 224, 224-232.

16 Y. J. Bai, J. D. Xu, M. Kong, Q. Gao, L. F. Liu and S. L. Li, Food Res. Int., 2015, 76, 387-394.

17 S. Y. Dai, Z. Z. Lin, B. Xu, Y. Q. Wang, X. Y. Shi, J. Y. Zhang and Y. J. Qiao, Talanta, 2018, 189, 641-648.

18 K. J. Karczewski and M. P. Snyder, Nat. Rev. Genet., 2018, 19, 299-310.

19 M. S. Kwon, Y. Kim, S. Lee, J. Namkung, T. Yun, S. G. Yi, S. Han, M. Kang, W. K. Sun and J. Y. Jang, BMC Genomics, 2015, 16, S4.

20 M. D. Ritchie, E. R. Holzinger, L. Ruowang, S. A. Pendergrass and K. Dokyoon, Nat. Rev. Genet., 2015, 16, 85-97.

21 K. Anshul, M. Wouter, E. Jason, B. Misha, Y. Angela, H.-M. Alireza, K. Pouya, Z. Zhizhuo, W. Jianrong and Z. Michael J, Nature, 2015, 518, 317-330.

22 Y. J. Bai, J. D. Xu, M. Kong, Q. Gao, L. F. Liu and S. L. Li, Food Res. Int., 2015, 76, 387-394.

23 W. Z. Yang, Y. B. Zhang, W. Y. Wu, L. Q. Huang, D. A. Guo and C. X. Liu, Acta Pharm. Sin. B, 2017, 7, 439-446. 
24 Y. Kashiwada, Y. Omichi, S. I. Kurimoto, H. Shibata, Y. Miyake, T. Kirimoto and Y. Takaishi, Phytochemistry, 2013, 96, 423-429.

25 Y. Yu, C. G. Zhu, S. J. Wang, W. X. Song, Y. C. Yang and S. J. Gong, J. Nat. Prod., 2013, 76, 2226-2233.

26 S. Qiu, W. Z. Yang, X. J. Shi, C. L. Yao, M. Yang, L. Xuan, B. H. Jiang, W. Y. Wu and D. A. Guo, Anal. Chim. Acta, 2015, 893, 65-76.

27 L. Y. Lin, P. P. Wang, Z. Y. Du, W. C. Wang, Q. F. Cong, C. P. Zheng, C. Jin, D. Kan and C. H. Shao, Int. J. Biol. Macromol., 2016, 88, 130-137.

28 M. H. Han, W. S. Lee, A. Nagappan, S. H. Hong, J. H. Jung, C. Park, H. J. Kim, G. Y. Kim, G. Kim and J. M. Jung, Phytother. Res., 2016, 30, 1824-1832.

29 Z. X. Liu, Z. Cheng, Q. J. He, B. Lin, P. Y. Gao, L. Z. Li, Q. B. Liu and S. J. Song, Fitoterapia, 2016, 110, 44-51.

30 C. Park, W. S. Lee, M. H. Han, K. S. Song, S. H. Hong, A. Nagappan, G. Y. Kim, G. S. Kim, J. M. Jung and C. H. Ryu, Phytother. Res., 2017, 32, 504-513.

31 X. Shang, H. Pan, M. Li, X. Miao and H. Ding, J. Ethnopharmacol., 2011, 138, 1-21.

32 Chinese Pharmacopoeia Commission, Chinese Pharmacopoeia, China Medical Science Press, Beijing, 2015, vol. 1.
33 S. Y. Dai, Z. P. Shang, F. Wang, Y. Cao, X. Y. Shi, Z. Z. Lin, Z. Wang, N. Li, J. Q. Lu and Y. J. Qiao, Sci. Rep., 2017, 7, 9971. 34 J. Y. Zhan, P. Yao, C. W. Bi, K. Y. Zheng, W. L. Zhang, J. P. Chen, T. T. Dong, Z. R. Su and K. W. Tsim, Phytomedicine, 2014, 21, 1318-1324.

35 A. Biancolillo, R. Bucci, A. L. Magrì, A. D. Magrì and F. Marini, Anal. Chim. Acta, 2014, 820, 23-31.

36 J. Y. Zhang, Z. J. Wang, Q. Zhang, F. Wang, Q. Ma, Z. Z. Lin, J. Q. Lu and Y. J. Qiao, Talanta, 2014, 124, 111-122.

37 J. Y. Zhang, Q. Zhang, N. Li, Z. J. Wang, J. Q. Lu and Y. J. Qiao, Talanta, 2013, 104, 1-9.

38 X. Sun, X. B. Cui, H. M. Wen, C. X. Shan, X. Z. Wang, A. Kang, C. Chai and W. Li, J. Pharm. Biomed. Anal., 2017, 141, 19-31.

39 M. Yang, Z. Zhou and D. A. Guo, Anal. Chim. Acta, 2015, 894, 44-53.

40 X. Zhang, Z. Ning, D. Ji, Y. Chen, C. Mao and T. Lu, J. Sep. Sci., 2015, 38, 3825-3831.

41 R. F. Goddu, in Near-infrared spectrophotometry, Advances in Analytical Chemistry and Instrumentation, ed. C. N. Reilly, 2nd edn, 1960, pp. 347-424.

42 S. Qiu, W. Z. Yang, X. J. Shi, C. L. Yao, M. Yang, X. Liu, B. H. Jiang, W. Y. Wu and D. A. Guo, Anal. Chim. Acta, 2015, 893, 65-76.

43 T. H. Wang, J. Zhang, X. H. Qiu, J. Q. Bai, Y. H. Gao and W. Xu, Molecules, 2015, 21, E40. 